\title{
Knowledge, attitude and practices of environmental health practitioners conducting food-borne disease outbreak investigation at a local municipality in Gauteng province, South Africa
}

\begin{tabular}{|c|c|}
\hline \multicolumn{2}{|c|}{$\begin{array}{l}\text { Authors: } \\
\text { Thokozani P. Mbonane }{ }^{1} \text { (D) } \\
\text { Nisha Naicker }{ }^{1,2,3}\end{array}$} \\
\hline \multicolumn{2}{|c|}{$\begin{array}{l}\text { Environmental Health, } \\
\text { Faculty of Health Sciences, } \\
\text { University of Johannesburg, } \\
\text { Johannesburg, South Africa }\end{array}$} \\
\hline \multicolumn{2}{|c|}{$\begin{array}{l}{ }^{2} \text { School of Public Health, } \\
\text { Faculty of Health Sciences, } \\
\text { University of the } \\
\text { Witwatersrand, } \\
\text { Johannesburg, South Africa }\end{array}$} \\
\hline \multicolumn{2}{|c|}{$\begin{array}{l}{ }^{3} \text { Epidemiology and } \\
\text { Surveillance Section, National } \\
\text { Institute for Occupational } \\
\text { Health, Johannesburg, } \\
\text { South Africa }\end{array}$} \\
\hline \multicolumn{2}{|c|}{$\begin{array}{l}\text { Research Project Registration: } \\
\text { Project Number: } \\
\text { HDC01-16-2014 }\end{array}$} \\
\hline \multicolumn{2}{|c|}{$\begin{array}{l}\text { Corresponding author: } \\
\text { Thokozani Mbonane, } \\
\text { tmbonane@uj.ac.za }\end{array}$} \\
\hline \multicolumn{2}{|c|}{$\begin{array}{l}\text { Received: } 05 \text { Aug. } 2019 \\
\text { Accepted: } 24 \text { Feb. } 2020 \\
\text { Published: } 29 \text { June } 2020\end{array}$} \\
\hline \multicolumn{2}{|c|}{$\begin{array}{l}\text { How to cite this article: } \\
\text { Mbonane, T.P. \& Naicker, N., } \\
\text { 2020, 'Knowledge, attitude } \\
\text { and practices of } \\
\text { environmental health } \\
\text { practitioners conducting } \\
\text { food-borne disease outbreak } \\
\text { investigation at a local } \\
\text { municipality in Gauteng } \\
\text { province, South Africa', } \\
\text { Health SA Gesondheid 25(0), } \\
\text { a1359. https://doi.org/ } \\
\text { 10.4102/hsag.v25i0.1359 }\end{array}$} \\
\hline \multicolumn{2}{|l|}{ Read online: } \\
\hline 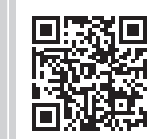 & $\begin{array}{l}\text { Scan this QR } \\
\text { code with your } \\
\text { smart phone or } \\
\text { mobile device } \\
\text { to read online. }\end{array}$ \\
\hline
\end{tabular}

Background: Food-borne disease (FBD) outbreaks are a common occurrence that is either not investigated or poorly investigated. According to anecdote evidence, this is because of nonuniformity to environmental health practices in South Africa.

Aim: This study aimed to determine and describe the knowledge, attitude and practices (KAP) of environmental health practitioners (EHPs) when conducting outbreak investigations of FBD at a local municipality.

Setting: This study was conducted in three sub-districts of Ekurhuleni Metropolitan Municipality (EMM), one of five municipalities in Gauteng province, South Africa.

Methods: A descriptive cross-sectional study was conducted to collect data using a semistructured questionnaire. Data collected were analysed using IBM Statistical Package for the Social Sciences. Continuous variables were compared using analysis of variances, and correlation was used to determine any association.

Results: Knowledge responses were scored out of 9. Open-ended questions were themed into five items (support, guidelines, resources, training and specialisation). Sixty-one (76.3\%) participants were randomly selected to participate in the study. There were more female participants (55.7\%) than male participants, and the mean age was 30.9 years. The participants' knowledge scores ranged from 1 to 9 . There were 17 (27.9\%) participants who have conducted FBD outbreak investigation. Twenty-six (42.6\%) believed that they were properly trained to conduct FBD outbreak investigations. Age was associated with position $(p=0.000)$ and qualification $(p=0.033)$.

Conclusion: The results indicated that there are gaps and challenges in the knowledge, while the practices were not consistent amongst environmental health practitioners. However, the attitude of EHPs was positive with regard to their role in FBD outbreak investigations.

Keywords: knowledge; attitude; practices; perceptions; food-borne disease; outbreaks and environmental health.

\section{Background}

Food-borne disease (FBD) outbreaks are a growing public health concern in developing countries, and are associated with high morbidity and mortality (Havelaar et al. 2015; Scott 2003). Foodborne diseases are linked to the consumption of food or water contaminated with toxins, viruses, bacteria or parasites, while FBDs occur where more than two persons have acquired the disease from the same contaminated food eaten at the same time (Dewey-Mattia et al. 2018; Jones et al. 2004). The occurrence of food-borne outbreaks is common, and the magnitude can be local, nationwide and international, thus requiring resources at all levels as well as numerous skills and knowledge (e.g. environmental health) to investigate (Ammon \& Tauxe 2007; Bélanger et al. 2015; Bisholo, Ghuman \& Haffejee 2018; Niehaus et al. 2011; Skovgaard 2009).

The prevalence of FBD and outbreaks is well known in developed countries compared to developing countries, where a lack of reporting is a major issue (Rocourt et al. 2003). The severity of FBD and outbreaks is relatively higher in developing and underdeveloped countries because of socio-economic and environmental factors (Broner et al. 2010; Newman et al. 2015). 
The populations at risk and those most likely to have a high mortality rate are infants, young children, the ageing and immunocompromised individuals (Rocourt 2013). Globally, it is estimated that 420000 deaths are associated with FBDs each year, while approximately 91 million persons get sick annually - 25000 being children under the age of 5 years (World Health Organization 2015).

Numerous FBD outbreaks are suspected to be poorly investigated and in some instances not investigated (World Health Organization 2008), especially in low- or middleincome countries because of the non-existence or poor implementation of surveillance programmes (Käferstein 2003). A qualitative study on the practices of environmental health specialists (refer to as environmental health practitioners [EHPs] in South Africa) during FBD outbreak investigation showed that practices amongst environmental health specialists were not consistent, and were hindered by numerous organisational and operational factors (Selman \& Green 2008). These included a lack of outbreak investigation training and experience, inadequate staff members, minimum or non-existent management support and poor collaboration between agencies (Selman \& Green 2008). These factors may lead to a poor outbreak investigation with the possibility of not identifying the environmental factors that contributed to the occurrence of an outbreak. Environmental health investigations can also be hindered by different factors such as unpreparedness, non-compliance with sampling procedure, poor reporting and delays in reporting (Department of Health Directorate: Food Control 2000).

In South Africa, a number of FBDs have occurred, and were reported and investigated in the past. However, the role of EHPs was not clearly defined in these reports (Shonhiwa et al. 2019). The environmental health scope of practice states that the role of an EHP is to identify environmental factors that could have contributed to the outbreaks (Coleman 2016; National Department of Health (NDoH), 2009). An environmental health investigation includes environmental, food and water sampling and traceback investigation. Furthermore, an EHP should collect environmental samples of all utensils that could have been used during the preparation and serving of the food implicated (Department of Health Directorate: Food Control 2000). Food samples are not always available, as either the food could have been all served or leftovers disposed of. In such a case, EHPs should review the production and distribution chain of the suspected food (Weiser et al. 2016). According to anecdotal and scientific evidence in South Africa, laboratory and epidemiological investigations are well organised, conducted and reported compared to environmental health investigations (Muvhali et al. 2017; Ntshiqa et al. 2016; Smith et al. 2019; Sigudu, Tint \& Archer 2015). Furthermore, little has been written about the actual procedures followed during environmental health investigations in South Africa, except guidelines written in 2010 (Department of Health Directorate: Food Control 2000). The aim of the study was to determine and describe the knowledge, attitudes and practices of EHPs concerning investigations of FBD outbreaks at a metropolitan municipality in Gauteng province, South Africa.

\section{Research methods and design Study design, site and population}

A descriptive cross-sectional study was conducted amongst EHPs registered with the Health Professions Council of South Africa (HPCSA) and employed at Ekurhuleni Metropolitan Municipality (EMM), one of five municipalities in Gauteng province, South Africa. The EHPs are stationed in three subdistrict regions (SDRs) of the municipality, namely, South SDR (Germiston), North SDR (Kempton Park) and East SDR (Springs), as shown in Figure 1. Each EHP at municipalities is responsible for rendering municipal health services (MHS) within their area of jurisdiction. These services include water quality monitoring, food control, waste management, health surveillance of premises, surveillance and prevention of communicable diseases (excluding immunisations), vector control, environmental pollution control, disposal of the dead and chemical safety (Balfour 2006). The researchers received permission from the Director of Municipal Health Services to access the list of EHPs in the municipality, and to approach them. At the time of conducting the study, there were 103 permanently employed EHPs, including supervisors and SDR managers servicing a population of 3178470 million (Municipalities of South Africa 2019). The sample size of 80 was calculated using a sample size calculator with a $95 \%$ confident interval, and a $5 \%$ error of margin for a known population. The EHPs were then grouped according to their SDR, and randomly selected. The structured questionnaires were sent to the area manager secretary's office for distribution. A total of 61 EHPs (response rate of $76 \%$ ) from 80 potential participants completed and returned the questionnaire.

\section{Data collection}

A semi-structured questionnaire was used to collect information on demographic data of the participants, knowledge on conducting food-borne outbreak investigations, attitude on conducting and improving the investigations, and current practice of EHPs when conducting outbreak investigations in the municipality. The participants were further asked to give recommendations to improve outbreak investigations. The questionnaire was designed in English, as the participants were deemed conversant because of their educational level required to register with the HPCSA as independent EHPs. Questionnaires were handed and distributed by the secretary of the each SDR manager, and were collected after 7 days. The questionnaire was developed using the literature, as there were no previous studies conducted on the topic at the time the study was conducted (as per the knowledge of the researcher). Furthermore, it was pre-tested prior to the actual study to ensure accuracy and reliability. Ten EHPs from the three SDRs were invited to partake in the pilot study, and they were excluded from the actual study. 


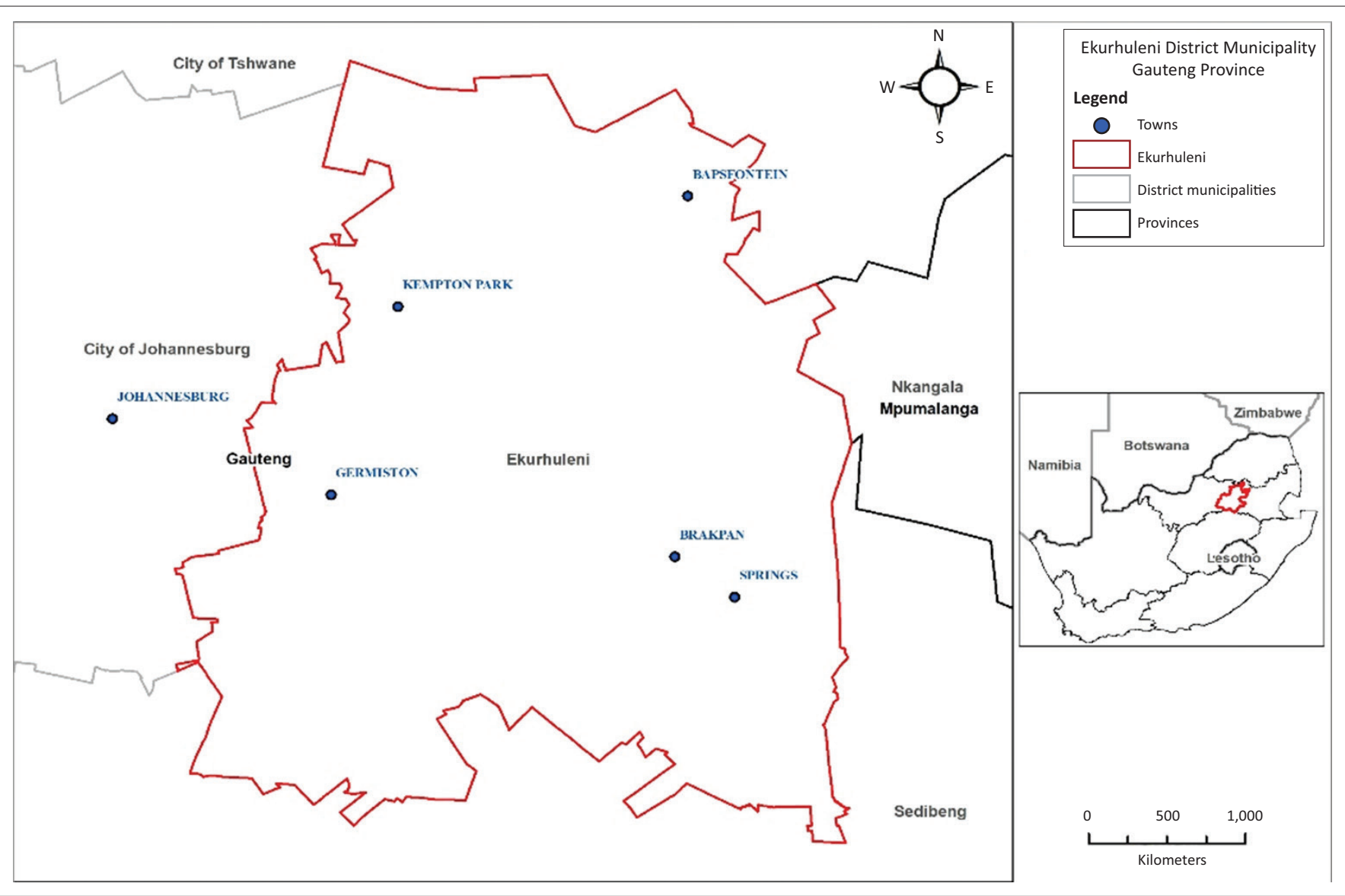

FIGURE 1: Map showing main towns of the subdistricts.

\section{Data management and analysis}

Data from questionnaires were entered and captured on Microsoft Excel for cleaning and sorting. Thereafter, data were transferred to IBM Statistical Package for the Social Sciences (SPSS) Statistics Version 25 for analysis. Participants were given one point for a correct response in the knowledge section, whose points were then totalled. The data on the other variables (attitude and practices) of interest were not scored. Responses on the recommendation were themed into four categories after participants responded: guidelines, resources, training and notification. Descriptive analyses such as frequency, mean, standard deviation and range were conducted. Analysis of variance (ANOVA) was used to compare means of continuous variables (demographic information). The association between knowledge score and demographic information was determined using correlation. The level of significance was set at 0.05 .

\section{Ethical consideration}

Ethical approval for the study was granted by the Faculty of Health Sciences' Academics Ethics Committee of the University of the Johannesburg. Permission to conduct the study was secured from the EMM. Participants were given an information sheet describing the aim and objectives of the study and signed written consent prior to taking part in the study. Furthermore, they were informed of voluntary participation, and the right to withdraw anytime without any punitive action. The ethics approval number for the study was AEC75-01-2013. Ethical clearance was sought from and granted by the University of Johannesburg, Faculty of Health Sciences, Research Ethics Committee (Ethical Clearance Number: AEC75-01-2013).

\section{Results}

From the 61 (76.3\% response rate) EHPs who volunteered to participate in the study, $34(55.7 \%)$ were female participants and 27 were male participants. Amongst these, 37.7\% $(n=23)$ were from South SDR, 34.4\% $(n=21)$ were stationed at East SDR and $27.9(n=17)$ were from North SDR. Participants held different positions, and the majority were at an operational level $(73.8 \%)$. The highest formal qualification obtained by one participant was a master's degree, 32 (52.5\%) participants had a BTech degree and 21 (34.4\%) participants had a national diploma, while others (4.9\%) had either an advanced diploma or honours degree in another field as an additional qualification (see more details on Table 1). The participants' age ranged from 23 to 53 years, while the mean $( \pm \mathrm{SD})$ was $30.9( \pm 7.8)$ years.

There was no statistical significance between the EHPs from the three subdistricts for gender $(p=0.451)$, age $(p=0.595)$, position $(p=0.200)$, years of experience $(p=0.733)$ and highest formal qualification $(p=0.378)$. Hence, the data 
were merged for further analysis (for correlation). In the merged data set using Pearson's correlation, a positive significant relationship between age and position $(r=0.544$; $p=0.000$ ) was found. Furthermore, there was a positive significant relationship between age and qualification $(r=0.237 ; p=0.033)$.

\section{Knowledge of food-borne disease outbreak investigations}

There were nine questions on knowledge relating to FBD outbreak investigations (see Table 2 for the detailed findings

\begin{tabular}{|c|c|c|}
\hline Demographic information & Frequency (no.) & Percentage \\
\hline \multicolumn{3}{|l|}{ Gender } \\
\hline Male & 27 & 44.3 \\
\hline Female & 34 & 55.7 \\
\hline \multicolumn{3}{|l|}{ Age } \\
\hline $21-25$ years & 14 & 23.0 \\
\hline $26-30$ years & 28 & 45.9 \\
\hline $31-35$ years & 7 & 11.5 \\
\hline $36-40$ years & 4 & 6.6 \\
\hline $41-45$ years & 2 & 3.3 \\
\hline 46 or older & 6 & 11.5 \\
\hline \multicolumn{3}{|l|}{ Subdistrict station or office } \\
\hline East & 21 & 34.4 \\
\hline South & 23 & 37.7 \\
\hline North & 17 & 27.9 \\
\hline \multicolumn{3}{|l|}{ Position } \\
\hline EHP & 45 & 73.8 \\
\hline Senior EHP & 14 & 23.0 \\
\hline Assistant Chief EHP & 2 & 3.3 \\
\hline \multicolumn{3}{|l|}{ Years of experience } \\
\hline $0-5$ years & 35 & 57.4 \\
\hline $6-10$ years & 14 & 23.0 \\
\hline $11-15$ years & 3 & 4.9 \\
\hline 16 years above & 9 & 14.8 \\
\hline \multicolumn{3}{|l|}{ Highest formal qualification } \\
\hline National diploma & 21 & 34.4 \\
\hline Advanced diploma & 4 & 6.6 \\
\hline BTech degree & 32 & 52.5 \\
\hline Masters & 1 & 1.6 \\
\hline Others & 3 & 4.9 \\
\hline
\end{tabular}

EHP, environmental health practitioner.

TABLE 2: Participants' responses to the knowledge questions.

\begin{tabular}{lcc}
\hline Question & Correct (\%) & Incorrect (\%) \\
\hline $\begin{array}{l}\text { How would you define a food-borne diseases } \\
\text { outbreak? }\end{array}$ & 24.6 & 75.4 \\
$\begin{array}{l}\text { What is the difference between food-borne disease } \\
\text { and food-borne diseases outbreak? }\end{array}$ & 13.1 & 86.9 \\
$\begin{array}{l}\text { Why is it important to carry out an immediate } \\
\text { environmental health field investigation? }\end{array}$ & 14.8 & 85.2 \\
$\begin{array}{l}\text { What would be your actions at a food-borne disease } \\
\text { outbreak site? }\end{array}$ & 16.4 & 83.6 \\
$\begin{array}{l}\text { What is the temperature at which food samples } \\
\text { should be kept at? }\end{array}$ & 18 & 82 \\
$\begin{array}{l}\text { How would you ensure that the chain of custody is } \\
\text { maintained after a food sample has been collected? }\end{array}$ & 31.1 & 68.9 \\
$\begin{array}{l}\text { What is a food traceback investigation? } \\
\begin{array}{l}\text { What type of samples will you take during a } \\
\text { food-borne disease outbreak? }\end{array}\end{array}$ & 52.5 & 47.5 \\
$\begin{array}{l}\text { What is the recommended time frame to complete } \\
\text { and submit a report on food-borne disease } \\
\text { according to current legislation? }\end{array}$ & 29.5 & 70.5 \\
\hline
\end{tabular}

on knowledge). Participants were asked to define 'foodborne disease outbreak'. Fifteen responded (24.6\%) provided the correct response, namely, that FBD outbreak is the occurrence of two or more cases of FBDs from the ingestion of a common food at the same time and place (Dewey-Mattia et al. 2018). Nine (14.8\%) gave a correct answer on the importance of conducting an environmental investigation (i.e. finding the environmental factors that contributed to the occurrence of FBD outbreaks and prevent future outbreak of similar nature). Only 10 (16.4\%) participants gave a correct response (interview those involved, take food and environmental samples, and environmental assessment of the facility at the site where an outbreak has occurred).

Questions on the adequate temperature $\left(-2{ }^{\circ} \mathrm{C}\right)$ at which samples must be kept were answered correctly by 11 (18.0) participants, compared to 50 (82.0) incorrect answers received. Nineteen $(31.1 \%)$ participants knew how to maintain a chain of custody (ensuring that there is evidence or a paper trail of handling samples safely from the source to the laboratory). Approximately half $(n=32 ; 52.5)$ of the participants knew how to conduct a food traceback investigation. Thirty-four (55.7\%) EHPs knew which type of samples (food and environmental, excluding stools) to collect during an investigation of food-related disease outbreak. The score out of the total (Skovgaard 2009) on the participants' FBD outbreak knowledge ranged from 1 to 9 , with a mean $( \pm$ SD) of $2.4( \pm 1.5)$ points. There was no correlation between the knowledge score with any demographic items.

\section{Environmental health practitioners' attitudes towards food-borne disease outbreaks}

Twenty-six (42.6\%) participants believed that they were properly trained to conduct FBD outbreaks. All participants agreed that EHPs have a role in reducing the spread of food poisoning cases. Fifty-seven (93.4\%) participants believed that environmental health investigation has an impact on an outbreak investigation. Most participants ( $n=49 ; 80.3 \%$ ) indicated that it is important to conduct follow-up and monitoring visits after an outbreak. More than half ( $n=35 ; 57.4 \%$ ) of the participants indicated that it is difficult to conduct investigations and follow-ups effectively while trying to meet all the functions of MHS. Thirty-eight (62.3\%) participants believed that specialising in environmental epidemiology is necessary for conducting an effective

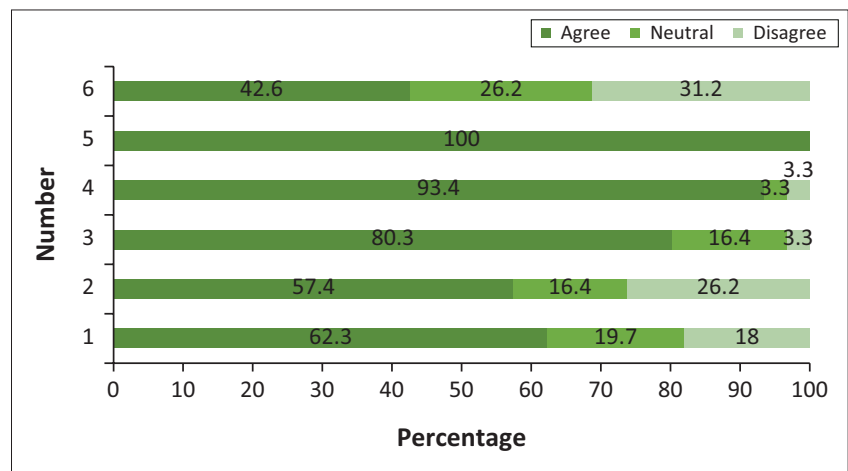

FIGURE 2: Environmental health practitioners' attitudes towards outbreak investigations. 
outbreak investigation. A detailed description of participants' attitudes is provided in Figure 2.

Using Pearson's correlation, a relationship between the statement 'I was properly trained to conduct investigations' was found with age $(r=0.262 ; p=0.041)$ and years of experience $(r=0.324 ; p=0.011)$. This showed that older participants and long-serving EHPs believed that they were properly trained.

\section{Practices of outbreak investigations}

Feedback on the participants' current practices when conducting outbreak investigations is summarised in Table 3. Seventeen $(27.9 \%)$ participants indicated that they have conducted an FBD outbreak investigation, compared to $44(72.1 \%)$. Forty $(65.6 \%)$ participants stated that they knew about an occurrence of an outbreak immediately when it had occurred. Thirteen $(21.3 \%)$ respondents stated that they knew about an outbreak a day after it has occurred, while five $(8.2 \%)$ participants knew after evidence (suspected food) was removed. Twenty-eight $(45.9 \%)$ participants took samples within the first $12 \mathrm{~h}$ after the outbreak has occurred, while $33(54.1 \%)$ participants took samples after $12 \mathrm{~h}$. The majority (91.8\%) of the participants submitted samples to the laboratory within $12 \mathrm{~h}$ after collecting them. More than half ( $n=31 ; 50.8 \%$ ) of the participants 'always' conducted followup or monitoring visits, and $21(34.4 \%)$ EHPs sometimes conducted these visits and nine $(14.8 \%)$ never conducted monitoring visits. Out of the 52 participants who either always or sometimes conducted follow-up visits, 20 (38.5\%) took 2 weeks to conduct these visits, 13 (25.0\%) a week, 12 $(23.1 \%)$ a month and seven $(13.5 \%)$ took more than a month. Approximately 20\% reviewed and requested documents (e.g. notification letters), 17 (27.9\%) called a relevant person and $52.5 \%$ either consulted their supervisor or colleagues. Six $(9.8 \%)$ EHPs prepared for outbreak investigation by arranging sampling equipment and liaised with the outbreak response team (ORT), while $11(18.0 \%)$ and $26(42.6 \%)$ only communicated with ORT and arranged sampling, respectively.

The results differed from the guidelines developed in 2010, which stipulates that EHPs should be informed immediately after an outbreak has occurred (Department of Health Directorate: Food Control 2000). This will allow them to confirm the occurrence of the outbreak by contacting the reporting facility and reviewing notifications. While in the office, they need to prepare for the investigation through gathering of materials needed and contacting the ORT if a need arises. Samples of the suspected food should be collected immediately and submitted to the laboratory for analysis. Furthermore, it is expected that EHPs conduct a follow-up visit to monitor implementation of the investigation's recommendations as per the guidelines (Department of Health Directorate: Food Control 2000).

Sixty (98.4\%) participants recommended that the municipality should develop a guideline document in a form of standard of the procedure (SOP). Approximately $69 \%$ of participants

TABLE 3: Practices in relation to outbreak investigations.

\begin{tabular}{|c|c|c|c|}
\hline Questions & Responses & Frequency (no.) & Percentage (\%) \\
\hline \multirow{2}{*}{$\begin{array}{l}\text { Have you been involved in a food-borne disease outbreak investigation } \\
\text { as an EHP? }\end{array}$} & No & 44 & 72.1 \\
\hline & Yes & 17 & 27.9 \\
\hline \multirow[t]{4}{*}{ When do you become aware of food-borne diseases outbreak? } & Immediately when it has occurred & 40 & 65.6 \\
\hline & A day after it has occurred & 13 & 21.3 \\
\hline & When all evidence has been removed & 5 & 8.2 \\
\hline & Other & 3 & 4.9 \\
\hline \multirow{2}{*}{$\begin{array}{l}\text { While in the office, how would you confirm or determine that an } \\
\text { outbreak may have occurred? }\end{array}$} & Calling relevant employees & 17 & 27.9 \\
\hline & Other & 32 & 52.5 \\
\hline \multirow[t]{4}{*}{ How do you prepare for an outbreak investigation before going out? } & Arrange sampling equipment & 26 & 42.6 \\
\hline & Communicate with ORT and arrange sampling equipment & 6 & 9.8 \\
\hline & Communicate with ORT & 11 & 18.0 \\
\hline & Other & 18 & 29.5 \\
\hline \multirow{4}{*}{$\begin{array}{l}\text { What is the appropriate time frame for taking a sample after an outbreak } \\
\text { has occurred? }\end{array}$} & Last $12 \mathrm{~h}$ & 28 & 45.9 \\
\hline & Last $48 \mathrm{~h}$ & 4 & 6.6 \\
\hline & Last $72 \mathrm{~h}$ & 3 & 4.9 \\
\hline & Other & 8 & 13.1 \\
\hline \multirow{2}{*}{$\begin{array}{l}\text { After collecting the food sample, how long on average does it take you to } \\
\text { submit the samples to the laboratory? }\end{array}$} & Within $12 \mathrm{~h}$ & 56 & 91.8 \\
\hline & Between 12 and $24 \mathrm{~h}$ & 5 & 8.2 \\
\hline \multirow{3}{*}{$\begin{array}{l}\text { How often do you conduct follow-up or monitoring visits after report } \\
\text { writing? }\end{array}$} & Never & 9 & 14.8 \\
\hline & Sometimes & 21 & 34.4 \\
\hline & Always & 31 & 50.8 \\
\hline \multirow{4}{*}{$\begin{array}{l}\text { How long after the investigations do you conduct a follow-up or } \\
\text { monitoring visit? }\end{array}$} & One week & 13 & 25.0 \\
\hline & Two weeks & 20 & 38.5 \\
\hline & One month & 12 & 23.1 \\
\hline & More than 1 month & 7 & 13.5 \\
\hline
\end{tabular}

ORT, outbreak response team; EHP, environmental health practitioner. 
indicated the need to provide adequate training in environmental epidemiology, and $57.4 \%(n=35)$ suggested that the municipality should introduce specialisation in the rendering of MHS. All the participants $(n=61 ; 100 \%)$ mentioned the provision of adequate resources to conduct a successful outbreak investigation, as well as a need for management support.

\section{Discussion}

This study demonstrated that there is inconsistency in terms of knowledge and practice of EHPs pertaining to outbreak investigations. According to Shonhiwa et al. (2019), there is a large degree of inconsistency in the reporting and investigation of FBDs throughout South Africa (Shonhiwa et al. 2019). However, this is not localised to South Africa. In the United States, only $57 \%$ of reported food-borne-related outbreaks were investigated properly (Krosnick 1999), and hence, the importance of determining the KAP on FBD environmental outbreak investigations amongst EHPs. This is the first study to determine KAP on FBDs outbreak investigation amongst EHPs in South Africa. The knowledge on outbreak investigations concepts was low. However, the attitude was positive and the practices were inconsistent. The EHP per population ratio was 1:30 859. This is too high when compared to the World Health Organization's recommendation (1 EHP: 10000 population) and the South African adopted standard of 1 EHP: 15000 population (Balfour 2006). This could obstruct the provision of Environmental Health Services (EHS) and its programmes - especially FBDs outbreak investigation - as highlighted by Shezi and co-authors (Shezi et al. 2019).

The knowledge score on FBDs and its outbreak investigations were poor. This was similar to other studies conducted amongst public health officials (Mathatha et al. 2018). The number of correct responses was very low $(88.6 \%$ scored less than four out of nine), and respondents responded as follows: differentiating between FBD and FBD outbreak $(13.1 \%)$, importance of conducting outbreak investigation $(14.8 \%)$, activities at the outbreak site $(16.4 \%)$, maintaining the cold chain $(18.0 \%)$, chain of custody $(31.1 \%)$ and time frame to finalise and provide feedback $(29.5 \%)$. This could impair on the urgency of conducting an outbreak investigation, as these differ from routine inspections (Petran, White \& Hedberg 2012). Such conduct might lead to failure in determining the environmental factors that contributed to the occurrence of an outbreak, and may have detrimental consequences such as failure to prevent further outbreaks.

Even though most participants had a BTech degree, more than $50 \%$ believed that they were not properly trained, or were not sure. This was mostly influence by age $(p=0.041)$ and the number of years as a practicing EHP ( $p=0$ 011). However, participants had a positive attitude towards their role in outbreak investigation and the impact of their investigations. This could mean that EHPs are willing to be trained to improve their skills and to contribute meaningfully to the outcomes of outbreak investigations. Previously, the environmental health curriculum was not similar in South African institutions that offered the course, especially at BTech degree level, where students branched to specialisation. This could have created this knowledge gap amongst EHPs. The recently approved and implemented curriculum sought to address that challenge. This intervention will, however, not assist EHPs who are already in the field. In this study, EHPs believed that performing all nine MHS hinders them from being effective and conducting follow-up visits. However, they believed that specialisation in the profession is a solution to this challenge. There is a need to investigate this further, as most health professions have specialised in rendering health services.

In South Africa, there are a few outbreaks related to food contamination reported. Environmental practitioners rely on the clinical or laboratory staff for FBD outbreak notification. This could be explained by the high number of EHPs (72.1\%) who had not conducted an outbreak investigation prior to taking part in the study. According to Bisholo and colleagues, South Africa does not have the capacity to stop and prevent FBD outbreaks. In addition, lack of standardisation and uniformity in the provision of EHS have been a concern for a long time in South Africa (Balfour 2006), hence the development and implementation of the National Environmental Health Policy in 2013 (Department of Health 2014). Out of the seven questions on the practice, none of the activities showed consistency amongst EHPs. These findings are similar to a study in 2008 which found significant dissimilarity in the practice amongst environmental health specialists conducting outbreak investigations (Selman \& Green 2008).

The participants recommended the provision of adequate resources for conducting outbreak investigations. This has been an ongoing challenge that was previously raised by other officials and researchers (Balfour 2006; Department of Health 2014; Department of Health Directorate: Food Control 2000; Nahman, Wise \& De Lange 2009; Wright, Mathee \& Oosthuizen 2013). Environmental health practitioners suggested the development of guidelines in a form of SOP on outbreak investigation that will be compulsory to follow; yet there are national guidelines established in 2010 (Department of Health Directorate: Food Control 2000). This could mean a low level of awareness about this document, or lack thereof amongst EHPs. Encouragingly, they highlighted a need for specialised training on environmental health outbreak investigation. Mathee and Wright (2013) believed that specialised training can improve special skills in EHS (Mathee \& Wright 2013), as EHPs are trained on an array of fields of public health. Furthermore, they called for specialisation within the EH profession, as most believed that this will enable EHPs to become experts, rather than trying to achieve all functions of EHS at once. They also highlighted a need for management support in EHS provision. Some of these recommendations (management support, specialised training and provision of resources) are similar to those 
highlighted in another study on other functions of EHS (Shezi et al. 2019).

Municipal health services are part of primary health care at local government level (Robertson 2016). One of the major roles of EHPs at local government level is to monitor, control and manage environmental factors contributing to the development of disease (Wright, Mathee \& Garland 2014). Considering the recent severe Listeriosis outbreak and the proposed approach (National Health Insurance) to health care in the country (Chersich et al. 2018; Robertson 2016), the public health system will benefit immensely from a wellfunctional environmental health system ensuring the elimination of preventable conditions (such as FBD). This will ensure that these conditions do not overburden the public health system and its funds.

\section{Limitations of the study}

One of the limitations of the study was that it was conducted in one municipality, and the sample size was small. The results cannot be generalised for the whole country. It is recommended that a similar study be conducted amongst EHPs from different provinces. However, it is important to consider that the Gauteng province is the most populated province in South Africa. Secondly, data were collected using a self-administered questionnaire, which could have introduced recall bias. While the study had its limitations, it can be used as a baseline to evaluate environmental or MHS, in particular, FBD outbreak investigation. Hence, the study findings contribute to the current body of knowledge and the significance of improving EHPs practices, especially with regard to the investigations of FBDs outbreaks. This will ensure that future outbreaks are adequately investigated, and that the future occurrence thereof can be prevented or limited.

\section{Recommendations}

The study recommends the development of an educational training programme to address the insufficient knowledge, as well as developing guidelines or SOP that will assist EHPs to prepare and conduct outbreak investigations. This will also address the concerns about non-standardised notification systems and improper and non-uniform reporting. The study can build on the positive attitude of participants through introducing specialisation. Lastly, the relevant municipality needs to ensure that EHPs responsible for outbreak investigations are provided with sufficient support from management and adequate resources.

\section{Conclusion}

The data in this study suggest that EHPs are not well conversant on how to conduct food-borne outbreak investigations. The current practices were not uniform and comprehensive. However, most participants recognise that there can be improvements in the current practices. Furthermore, the attitudes of participants were positive, even though there were concerning areas where participants were undecided.

\section{Acknowledgements}

The authors would like to thank Ms Bonita Allies, Mr. Themba Sigudu and Prof. Lucille Blumberg for contribution on a previous work related to the article, and Ms. Thandi Kapwata for designing the map. They would also like to thank Gauteng Department of Health for financial support towards the main author's educational fees. They also thank the municipality for granting permission to conduct the study and EHPs who participated in the study as well as all public health officials involved in FBDs outbreak investigation.

\section{Competing interests}

The authors declare that no conflicts of interest exist.

\section{Authors' contributions}

T.P.M. conducted data collection and prepared the article. N.N. reviewed and finalised the article.

\section{Funding information}

This research received no specific grant from any funding agency in the public, commercial or not-for-profit sectors.

\section{Data availability statement}

Data sharing is not applicable to this article as no new data were created or analysed in this study.

\section{Disclaimer}

The views and opinions expressed in this article are those of the authors and do not necessarily reflect the official policy or position of any affiliated agency of the authors.

\section{References}

Ammon, A. \& Tauxe, R.V., 2007, 'Investigation of multi-national foodborne outbreaks in Europe: Some challenges remain', Epidemiology and Infection 135(6), 887-889. https://doi.org/10.1017/S0950268807008898.

Balfour, T., 2006, Municipal health services in South Africa, opportunities and challenges, DbsaOrg, pp. 1-12, viewed 20 May 2019, from http://www.dbsa. org/Research/Documents/MunicipalhealthservicesinSouthAfricaOpportunities andchallenges.pdf.

Bélanger, P., Tanguay, F., Hamel, M. \& Phypers, M., 2015, 'An overview of foodborne outbreaks in Canada reported through outbreak summaries: 2008-2014', The Canada Communicable Disease Report 41(11), 254-262. https:// doi.org/10.14745/ccdr.v41i11a01

Bisholo, K.Z., Ghuman, S. \& Haffejee, F., 2018, 'Food-borne disease prevalence in rural villages in the Eastern Cape, South Africa', The African Journal of Primary Health \& Family Medicine 10(1), e1-e5. https://doi.org/10.4102/phcfm.v10i1.1796

Broner, S., Torner, N., Dominguez, A., Martínez, A. \& Godoy, P., 2010 'Sociodemographic inequalities and outbreaks of foodborne diseases: An ecologic study', Food Control 21(6), 947-951. https://doi.org/10.1016/j. foodcont.2009.12.002

Chersich, M., Scorgie, F., Rees, H. \& Wright, C., 2018, 'How climate change can fuel listeriosis outbreaks in South Africa', South African Medical Journal 108(6), 453-454. https://doi.org/10.7196/SAMJ.2018.v108i6.13274

Coleman, E.W., 2016, 'Equipping environmental health workers with environmental assessment tools', Journal of Environmental Health 78(1), 36-38, viewed n.d., from http://www.ncbi.nlm.nih.gov/pubmed/4620656.

Department of Health, 2014, National environmental health policy, pp. 1-132, Government Gazette, viewed 05 February 2015, from http://www.greengazette. co.za/pages/national-gazette-37230-of-17-january-2014-vol-583_20140117 GGN-37230-003.

Department of Health Directorate: Food Control, 2000, Guidelines for an environmental health officer (EHO) engaged in food poisoning investigations, Government Gazette, Pretoria, viewed 04 February 2015, from http://www.doh. gov.za/docs. 
Dewey-Mattia, D., Manikonda, K., Hall, A.J., Wise, M.E. \& Crowe, S.J., 2018 , 'Surveillance for foodborne disease outbreaks - United States, 2009-2015', MMWR Surveillance Summaries 67(10), 1-11. https://doi.org/10.15585/mmwr. Ss6710a1

Havelaar, A.H., Kirk, M.D., Torgerson, P.R., Gibb, H.J., Hald, T., Lake, R.J. et al., 2015, 'World Health Organization global estimates and regional comparisons of the burden of foodborne disease in 2010', PLoS Medicine 12(12), e1001923. https:// doi.org/10.1371/journal.pmed.1001923

Jones, T.F.F., Imhoff, B., Samuel, M., Mshar, P., McCombs, K.G.G., Hawkins, M. et al., 2004, 'Limitations to successful investigation and reporting of foodborne outbreaks: An analysis of foodborne disease outbreaks in FoodNet catchment areas, 1998-1999', Clinical Infectious Diseases 38(s3), S297-S302. https://doi. org/10.1086/381599

Käferstein, F., 2003, 'Foodborne diseases in developing countries: Aetiology, epidemiology and strategies for prevention', International Journal of org/10.1080/0960312031000102949

Krosnick, J.A., 1999, 'Survey research', Annual Review of Psychology 50(3), 537-567. https://doi.org/10.1146/annurev.psych.50.1.537

Mathatha, E.D., Manamela, J.M., Musekiwa, A. \& Prabdial-Sing, N., 2018, 'Exploring the knowledge, attitudes and practices (KAP) of health care professionals on vira hepatitis notification in Gauteng, South Africa, 2015', Archives of Public Health 76(1), 75. https://doi.org/10.1186/s13690-018-0319-8

Mathee, A. \& Wright, C.Y., 2013, 'Environmental health in South Africa', in A. Padarath \& R. English (eds.), pp. 105-116, Health System Trust, Durban, viewed 20 May 2019, from https://www.hst.org.za/publications/SouthAfricanHealthRev ews/10EnvironmentalHealthinSouthAfrica-SAHR2014. pdf\#search=mathee.

Municipalities of South Africa, 2019, Ekurhuleni Metropolitan municpalilty, viewed 05 June 2019, from https://municipalities.co.za/demographic/4/city-of-ekurhulenimetropolitan-municipality.

Muvhali, M., Smith, A.M., Rakgantso, A.M. \& Keddy, K.H., 2017, 'Investigation of Salmonella Enteritidis outbreaks in South Africa using multi-locus variable-
number tandem-repeats analysis, 2013-2015', BMC Infectious Diseases 17(1), 661. https://doi.org/10.1186/s12879-017-2751-8

Nahman, A., Wise, R. \& De Lange, W., 2009, 'Environmental and resource economies in South Africa: Status quo and lessons for developing countries', South African in South Africa: Status quo and lessons for developing countries', South African
Journal of Science 105(9-10), 350-355. https://doi.org/10.4102/sajs.v105i9/ Journal

National Department of Health (NDoH), 2009, Regulations defining the scope of the profession of environmental: Amendment, R698, pp. 3-13, viewed 04 February 2015, from https://www.polity.org.za/article/health-professions-act-1974-regulationsdefining-the-scope-of-the-profession-of-environmental-health-amendmentgazette-no-32334---regulation-698-2009-07-02.

Newman, K.L., Leon, J.S., Rebolledo, P.A. \& Scallan, E., 2015, 'The impact of socioeconomic status on foodborne illness in high-income countries: A systematic review', Epidemiology and Infection 143(12), 2473-2485. https://doi.org/10.1017/ S0950268814003847

Niehaus, A.J., Apalata, T., Coovadia, Y.M., Smith, A.M. \& Moodley, P., 2011, 'An outbreak of foodborne salmonellosis in rural KwaZulu-Natal, South Africa', fpd.2010.0749

Ntshiqa, T., Mpangane, H., Mpambane, D. \& Moshime, M., 2016, 'Staphylococcal foodborne illness outbreak, Tshwane District, Gauteng Province - South Africa, June 2015', International Journal of Infectious Diseases 45(Suppl 1), 235. https:// doi.org/10.1016/j.ijid.2016.02.528

Petran, R.L., White, B.W. \& Hedberg, C.W., 2012, 'Health department inspection criteria more likely to be associated with outbreak restaurants in Minnesota' Journal of Food Protection 75(11), 2007-2015. https://doi.org/10.4315/0362 028X.JFP-12-148
Robertson, L.J., 2016, 'The South African Society of psychiatrists' response to the White Paper for National Health Insurance in South Africa', South African Journal of Psychiatry 22(1), 1053. https://doi.org/10.4102/sajpsychiatry. v22i1.1053

Rocourt, J., 2013, 'Foodborne diseases : Foodborne diseases and vulnerable groups', in Y. Motarjemi (ed.), Encyclopedia of food safety, vol. 1, pp. 323-331, Elsevier http://doi.org/10.1016/B978-0-12-378612-8.00080-9

Rocourt, J., Moy, G., Vierk, K. \& Schlundt, J., 2003, The present state of foodborne disease in $O E C D$ countries, WHO Library Cataloguing-in-Publication Data, pp. 23-43, viewed 12 June 2019, from http://www.who.int/foodsafety.

Scott, E., 2003, 'Food safety and foodborne disease in 21st century homes', Canadian JournalofinfectiousDisease 14(5),277-280. https://doi.org/10.1155/2003/363984

Selman, C.A. \& Green, L.R., 2008, 'Environmental health specialists' self-reported foodborne illness outbreak investigation practices', Journal of Environmental Health 70(6), 16-21; quiz 53-54, viewed n.d., from http://www.ncbi.nlm.nih.gov/ pubmed/18236932.

Shezi, B., Mathee, A., Siziba, W., Street, R.A., Naicker, N., Kunene, Z. et al., 2019 , 'Environmental health practitioners potentially play a key role in helping communities adapt to climate change', BMC Public Health 19(1), 54. https://doi. org/10.1186/s12889-018-6378-5

Shonhiwa, A.M. Ntshoe, G., Essel, V., Thomas, J. \& McCarthy, K, 2019, 'A review of foodborne diseases outbreaks reported to the outbreak response unit, national institute for communicable diseases, South Africa, 2013-2017', Internationa Journal of Infectious Diseases 79(1), 73. https://doi.org/10.1016/j.ijid.2018. 11.186

Sigudu, T.T., Tint, K.S. \& Archer, B., 2015, 'Epidemiological description of cholera outbreak in Mpumalanga Province, South Africa, December 2008-March 2009', Southern African Journal of Infectious Diseases 30(4), 125-128. http://doi.org/10. 1080/23120053.2015.1107263

Skovgaard, N., 2009, 'Foodborne disease outbreaks, guidelines for investigation and control', International Journal of Food Microbiology 135(2), 184-185. https://doi. org/10.1016/j.ijfoodmicro.2009.07.028

Smith, A.M., Tau, N.P., Smouse, S.L., Allam, M., Ismail, A., Ramalwa, N.R. et al., 2019 'Outbreak of Listeria monocytogenes in South Africa, 2017-2018: Laboratory activities and experiences associated with whole-genome sequencing analysis of isolates', Foodborne Pathogens and Disease 16(7), 524-530. https://doi. org/10.1089/fpd.2018.2586

Weiser, A.A., Thöns, C., Filter, M., Falenski, A., Appel, B. \& Käsbohrer, A., 2016, 'FoodChain-Lab: A trace-back and trace-forward tool developed and applied during food-borne disease outbreak investigations in Germany and Europe', PLoS One 11(3), e0151977. https://doi.org/10.1371/journal.pone.0151977

World Health Organization (WHO), 2008, Foodborne disease outbreak: Guidelines for investigation and control, WHO Press, Geneva, p. 146, viewed 01 February 2015, from https://www.who.int/foodsafety/publications/foodborne_disease/outbreak guidelines.pdf.

World Health Organization (WHO), 2015, Who estimates of the global burden of foodborne diseases, Foodborne Disase Burden Epidemiology Reference Group, viewed 11 June 2019, from https://www.who.int/foodsafety/publications/ foodborne_disease/fergreport/en/.

Wright, C.Y., Mathee, A. \& Garland, R.M., 2014, 'Climate change, human health and the role of environmental health practitioners', South African Medical Journal 104(8), 518-519. https://doi.org/10.7196/samj.7994

Wright, C.Y., Mathee, A. \& Oosthuizen, M.A., 2013, 'Challenging times for environmental health in South Africa: The role of the Environmental Health Research Network', South African Medical Journal 104(1), 20-21. https://doi. org/10.7196/samj.7287 